\title{
Placenta accreta in a referred post hysterotomy case: a bold step into the unknown
}

\author{
Shrinivas N. Gadappa, Rupali A. Gaikwad, Anurag A. Sonawne, \\ Ankita R. Shah, Shrutika O. Makde*, Sowjanya Shivanreddy Bheemareddy
}

Department of Obstetrics and Gynecology, Government Medical College, Aurangabad, Maharashtra, India

Received: 26 August 2020

Revised: 14 January 2021

Accepted: 15 January 2021

\author{
*Correspondence: \\ Dr. Shrutika O. Makde, \\ E-mail: shrutikamakde@gmail.com
}

Copyright: (c) the author(s), publisher and licensee Medip Academy. This is an open-access article distributed under the terms of the Creative Commons Attribution Non-Commercial License, which permits unrestricted non-commercial use, distribution, and reproduction in any medium, provided the original work is properly cited.

\begin{abstract}
Placenta accreta spectrum encompasses a range of pathological adherence of placenta and causes significant maternal and neonatal morbidity and mortality. With the increase in the number of cesarean deliveries over the last few decades, there has been an 8 fold increase in the incidence of placenta accreta. The single most important risk factor reported in about half the cases of PAS disorders is placenta previa. Management involves a standardized approach with a comprehensive multidisciplinary care team accustomed to management of placenta accreta. We discuss a rare case of a patient who underwent hysterotomy in an outside hospital and was referred to our tertiary care centre as atonic PPH. Undiagnosed antenatally and at the time of hysterotomy, she was diagnosed as a case of placenta accreta on exploration at our institute and was surgically managed.
\end{abstract}

Keywords: Placenta accreta, PPH, Uterine tamponade, Massive transfusion protocol

\section{INTRODUCTION}

Placenta accreta spectrum encompasses a range of pathological adherence of placenta and causes significant maternal and neonatal morbidity. The FIGO 2018 guideline has modified the current hypothesis as a defect in the endometrium-myometrium interface, typically at the site of prior hysterotomy that leads to a failure of normal decidualization in the corresponding uterine areas and allows trophoblastic infiltration within the myometrium and sometimes into surrounding pelvic organs. ${ }^{1}$

The major risk factors for placenta accreta spectrum are history of accreta in a previous pregnancy, previous caesarean delivery and other uterine surgery, including repeated endometrial curettage. ${ }^{2}$ The rate of PAS disorders have been seen to increase by $30 \%$ among women with repeated cesarean delivery. Compared with women with a primary cesarean delivery, women who underwent repeat cesarean were 2.13 times more likely to have PAS disorder. ${ }^{3}$ Ultrasound is highly accurate in diagnosing placenta accreta spectrum with a sensitivity of $89.5 \%$, a positive predictive value of $68 \%$ and a negative predictive value of $98 \%$. MRI may be used to complement ultrasound imaging to assess the depth of invasion and lateral extension of myometrial invasion. Accuracy of MRI for prediction of placenta accreta spectrum is good with a sensitivity of $75-100 \%$ and a specificity of $65-100 \%$. The use of color flow Doppler may facilitate diagnosis; turbulent lacunar flow being the most common finding in PAS disorders. ${ }^{2}$ Massive obstetric hemorrhage is the most common and potentially avoidable cause of maternal death whose management involves a standardized approach with a comprehensive multidisciplinary care team. ${ }^{1}$ 
Here we report a rare case of adherent placenta who was referred to our tertiary care centre by a gynecologist as a case of atonic PPH post hysterotomy, where adherent placenta was missed preoperatively in USG and intraoperatively during hysterotomy and was discovered while surgical management of $\mathrm{PPH}$, after extensive dissection of adhesions at lower uterine segment at our institute. She was successfully managed by timely referral, effective PPH management and obstetric hysterectomy.

\section{CASE REPORT}

This case report discussed a 29 years old, P2L2A1, resident of Beed district, Maharashtra, India who was brought to OBGY casualty by relatives and accompanying doctor on 25 July 2020. She was referred to $\mathrm{GMCH}$ from private hospital in view of atonic $\mathrm{PPH}$.

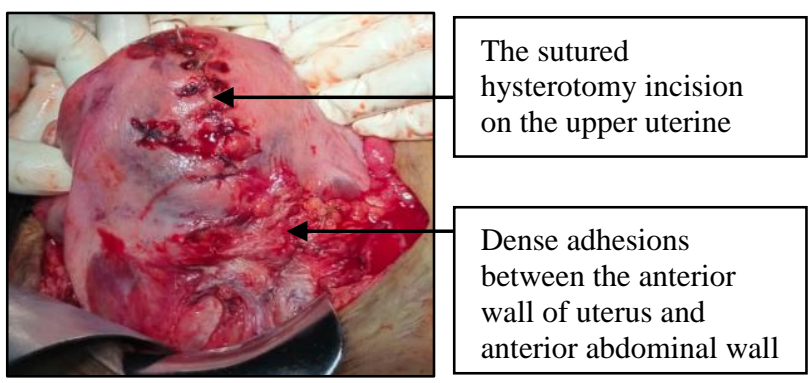

Figure 1: The evidence of sutured hysterotomy incision and dense adhesions at lower uterine segment.

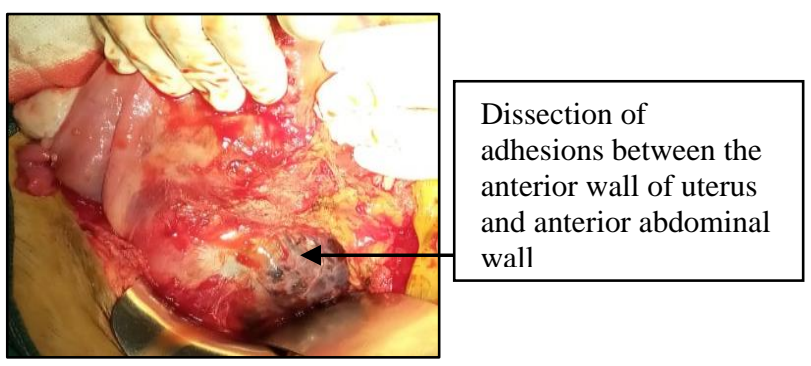

Figure 2: The dense adhesions between anterior abdominal and and anterior wall of uterus after dissection.

Patient G3P2L2 at 20 weeks gestation age with previous 2 lower segment cesarean section (LSCS) was admitted to private hospital with complaint of bleeding PV on 24 July 2020. Bleeding was painless, profuse, with soakage of up to 5 pads associated with passage of clots. Patient was admitted, transfused with 1 PRBC and posted for hysterotomy in view of placenta previa in bleeding phase on 24 July 2020. She was diagnosed as a case of atonic PPH 12 hours post operatively which was managed by insertion of Foleys catheter no 24 as uterine tamponade, transfused with $3 \mathrm{PRBC}$ and referred to $\mathrm{GMCH}$, Aurangabad. Obstetric ultrasonography done at 17 weeks revealed placenta previa completely covering internal os, i.e. placenta previa.
Patients' first LSCS was 6 years back which was uneventful, followed by second LSCS, 9 months back during which she was transfused 2 PRBC intraoperatively. On arriving at maternity casualty, the patient was having a pulse rate of 130/minute with blood pressure of $90 / 60 \mathrm{mmHg}$ and shock index of 1.44, the uterus being 22 weeks. She had a vaginal pack which was minimally soaked and a Foley's uterine tamponade in situ.

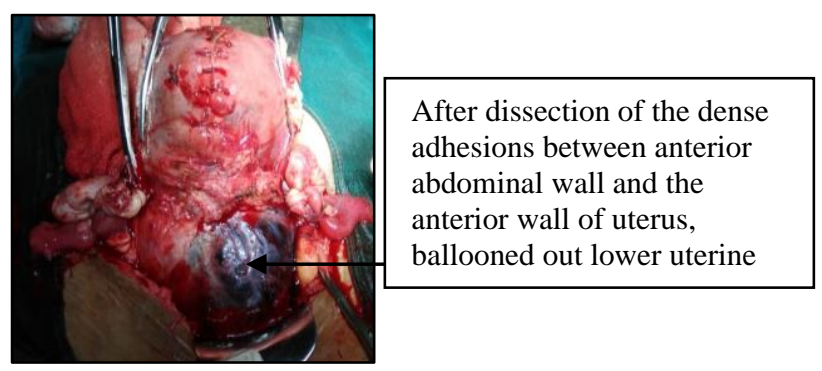

Figure 3: The ballooned out lower uterine segment and dense bluish vascularity suggestive of placenta accreta.

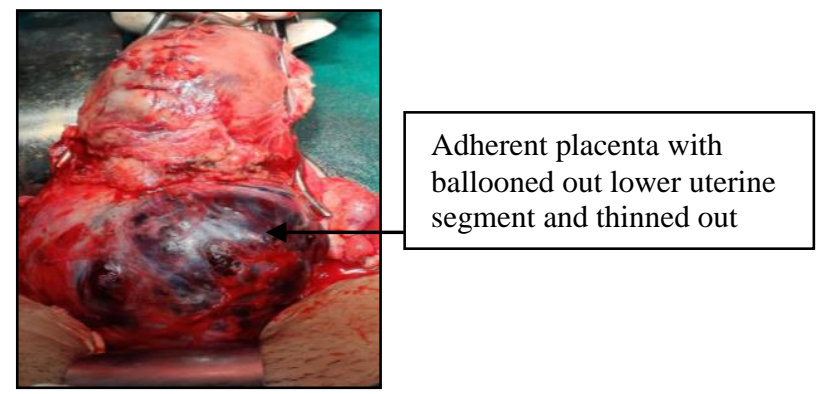

Figure 4: Adherent placenta with thinned out myometrium.

Immediate fluid and blood resuscitation was started and massive transfusion protocol was activated. Patient was posted for vaginal exploration SOS exploratory laprotomy SOS obstetric hysterectomy.

In OT under general anesthesia, on vaginal exploration, the vaginal pack and the Foley's uterine tamponade was removed after which there e/o passage of 100 grams of clots with active was bleeding per vaginum. Exploration of cervix and vagina was done which did not reveal any trauma. Decision of exploratory laprotomy was taken. The intraoperative findings were as follows; evidence of $100 \mathrm{ml}$ hemoperitoneum. On exploration only the sutured hysterotomy incision on upper uterine segment could be visualized due to dense adhesions between the lower uterine segment and the anterior abdominal wall.

After careful dissection of dense adhesions between the anterior abdominal wall, the advanced bladder and the lower uterine segment, ballooned out lower uterine segment was visualized which showed dense bluish vascularity suggestive of placenta accreta. With these 
intraoperative findings decision of obstetric hysterectomy was taken in view of adherent placenta.

Intraoperative blood transfusion included 4 PRBC, 4 FFP, and 4 RDP. Post operatively, patient was shifted to ICU, on ventilator and her vitals were strictly monitored. She was extubated 12 hours later, was vitally stable, eventually shifted to ward and was started on higher antibiotics. Her sutures were removed and she was discharged on post operative day 10.

\section{DISCUSSION}

The combination of placenta previa and placenta accreta is not a common occurrence in obstetrics, and it is one of the most disastrous complications encountered during surgery, especially when it is undiagnosed before surgery. ${ }^{4}$ For a woman with placenta previa the risk of associated placenta accreta is $3 \%, 11 \%, 40 \%, 60 \%$ for the first, second, third, fourth cesarean section respectively. ${ }^{2}$ With the availability of grey scale USG, MRI and Doppler, prenatal diagnosis of placenta accreta, placental localization and bladder invasion can be ruled out.

A patient with high risk factors for placenta accreta, presenting with bleeding from lower uterine segment raises a high index of suspicion and every attempt should be made to diagnose placenta accreta. The use of MRI in such suspicious cases is highly recommended. ${ }^{4}$ In the above discussed case, placenta accreta was undiagnosed in ANC period on USG and even at the time of surgery. During hysterotomy due to presence of dense adhesions between the anterior abdominal wall, the advanced bladder and anterior wall of uterus, the lower segment which had adherent placenta was not explored, thus posing a great risk to the mother's life.

The patient thus had PPH post operatively due the retained adherent placenta in situ and referred as atonic PPH. As she was at a low resource facility without blood bank at their disposal, rapid stabilization of patient in anticipation to transfer to higher facility was done. ${ }^{3}$ Uterine tamponade was used as a safe, feasible and effective measure to minimize the blood loss which helped her transport to higher facility for prompt management thus saving her life and decreasing her co morbidities. $^{5}$

Massive transfusion protocol was activated as a response to early signs of shock. Recent experiences in trauma cases suggest early correction of coagulopathy leads to improved outcome. Early administration of plasma, platelets, $\mathrm{RBC}$ in the defined ratio of $1: 1: 1$ is designed to avoid dilutional coagulopathy that may result from overtransfusion of one product. $^{6}$

The surgical management of placenta accreta with placenta previa include non-conservative such as subtotal or total hysterectomy and conservative, uterus sparing surgery such as placental resection or leaving the whole placenta in situ with or without planned interval hysterectomy. ${ }^{6}$ Management by a multidisciplinary team and delivery in a tertiary care facility improves outcome and lowers complication rates. ${ }^{7}$

Though timely blood transfusion, use of uterine tamponade, transfer of patient to facility equipped with a well equipped team, prompt and bold decision of exploration followed by obstetric hysterectomy and intensive follow up care help save the mother and give an excellent post operative outcome. ${ }^{8}$

\section{CONCLUSION}

Maternal mortality for undiagnosed case of placenta accreta is around 7\%. A case of previous 2 LSCS with placenta previa raises a high index of suspicion for placenta accreta. Antenatal diagnosis should be done with grey scale USG. MRI should be done in patients in whom the grey scale USG gives inconclusive result. In such cases of bleeding per vaginum in whom lower segment could not be visualized intraoperatively, active efforts should be done to visualize the lower uterine segment by dissecting the dense adhesions between the anterior abdominal wall and the anterior wall of uterus to rule out placenta accreta. When post op the patient was referred as atonic $\mathrm{PPH}$, the Foleys uterine tamponade and the urgent blood supply done helped gain a window for the transfer of the patient to a well equipped facility with highly trained obstetricians. Prompt surgical management, immediate resuscitation and extreme post operative care helped recovery of this patient.

Funding: No funding sources Conflict of interest: None declared Ethical approval: Not required

\section{REFERENCES}

1. Allen L, Jauniaux E, Hobson S, Papillon-Smith J, Belfort MA, et al. FIGO consensus guidelines on placenta accreta spectrum disorders: Nonconservative surgical management. Int $\mathbf{J}$ Gynaecol Obstet. 2018;140(3):281-90.

2. American College of Obstetricians and Gynecologists; Society for Maternal-Fetal Medicine. Obstetric Care Consensus No. 7: Placenta Accreta Spectrum. Obstet Gynecol. 2018;132(6):e259-75.

3. Creanga AA, Bateman BT, Butwick AJ, Raleigh L, Maeda A, Kuklina E, et al. Morbidity associated with cesarean delivery in the United States: is placenta accreta an increasingly important contributor?. Am J Obstet Gynecol. 2015;213(3):384.e1-11.

4. Okunowo AA, Ohazurike EO, Habeebu-Adeyemi FM. Undiagnosed placenta praevia percreta: A rare case report and review of management. Niger Postgrad Med J. 2019;26:61-4

5. Pala Ş, Atilgan R, Başpınar M, Kavak EÇ, Yavuzkır Ş, Akyol A, et al. Comparison of results of Bakri balloon tamponade and caesarean hysterectomy in 
management of placenta accreta and increta: a retrospective study. J Obstet Gynaecol. 2018;38(2):194-9.

6. Gatta LA, Lockhart EL, James AH. Blood products in the management of abnormal placentation. Clin obstet gynecol. 2018;61(4):828-40.

7. Sharma V, Kaur J. Case report on placenta accreta presenting obstetric emergency. Int $\mathrm{J}$ Reprod Contracept Obstet Gynecol. 2016;5(9):3261-4.

8. Gadappa S, Deshpande S, Rajpurohit K, Kalyankar B. Placenta percreta-an audacious experience. Int J
Reprod Contracept Obstet Gynecol. 2017;6(5):21124.

Cite this article as: Gadappa SN, Gaikwad RA, Sonawne AA, Shah AR, Makde SO, Bheemareddy SS. Placenta accreta in a referred post hysterotomy case: a bold step into the unknown. Int J Reprod Contracept Obstet Gynecol 2021;10:765-8. 